\title{
Hydrogen Effects on Material Performance
}

\author{
SAMANTHA K. LAWRENCE ${ }^{1,3}$ and JANELLE P. WHARRY ${ }^{2}$ \\ 1.-Los Alamos National Laboratory, Los Alamos, USA. 2.-Purdue University, West Lafayette, USA \\ 3.-e-mail: slawrence@lanl.gov
}

Numerous energy generation and transportation systems constructed of high-performance metal alloys are routinely exposed to hydrogen. The integrity of these systems is often challenged by a variety of hydrogen degradation modes affecting both structural components and storage media. In terms of hydrogen storage in metal vessels, two critical issues are the adsorption/desorption and permeation of hydrogen into the metal or alloy, as well as the potential for hydrogen embrittlement of the metal. ${ }^{1}$ In particular, many ferritic steels (typically used for pressure vessels), austenitic steels (typically used for dispensing or balance-of-plant applications) and nickel-base alloys exhibit a ductility minimum near room temperature, which is a serious concern for service, for example, as storage components in automotive applications. ${ }^{2,3}$ Degradation in these materials is often manifest by cracking, which compromises structural integrity, and depends on operating conditions, such as stress, temperature, and hydrogen pressure. For example, a modern steel exposed to environmental conditions (i.e., stress, cyclic loads, hydrogen pressure) typical of those found in pressure vessels and transport pipelines may crack at one-tenth of the design fracture toughness. ${ }^{4}$ Such degradation is generically termed "hydrogen embrittlement", but growing understanding of the mechanisms involved in hydrogen-materials interactions have evolved more precise terms for the various degradation modes observed in structural metals, including hydrogen environment-assisted cracking (HEAC), hydrogeninduced cracking (HIC), and internal hydrogenassisted cracking (IHAC). Generally, HEAC refers to the interaction of deformation/mechanical stress with atomic hydrogen, which is adsorbed from $\mathrm{H}_{2}$, hydrogen-bearing gasses (e.g., $\mathrm{H}_{2} \mathrm{O}, \mathrm{H}_{2} \mathrm{~S}$ ), or a hydrogen-bearing electrolyte. In this degradation mode, hydrogen-deformation interactions are often

Samantha K. Lawrence and Janelle P. Wharry are the JOM advisors for the Nanomechanical Materials Behavior Committee of TMS, and guest editors for the special topic Hydrogen Effects on Material Performance in this issue. localized in areas of high stress, such as crack tips and fracture process zones. HIC is also promoted by hydrogen adsorption in hydrogen sulfide-rich environments, ${ }^{5}$ and occurs by the generation and propagation of internal cracks, following hydrogen diffusion towards specific sites of the microstructure, including ferrite/pearlite bands and elongated MnS-type inclusions, where its accumulation and recombination into hydrogen gas causes internal pressures of several hundred bars, enough to crack the steel core (HIC) and blister the surface. ${ }^{6}$ Whereas HEAC and HIC are the result of hydrogen ingress into a metal during service, IHAC refers to atomic hydrogen dissolved during manufacturing, assembly, or environment exposure, and reduces strength or cracking resistance when mechanical loading and hydrogen uptake are sequential. ${ }^{4}$

Accompanying the growing understanding and vocabulary to describe hydrogen-assisted cracking phenomena is a refinement in understanding of fundamental hydrogen-material interactions, which influence materials performance in hydrogen environments. A considerable number of research efforts have identified hydrogen-metal interactions that contribute to degradation, including the formation of hydrides, ${ }^{7}$ hydrogen adsorption-induced dislocation emission, ${ }^{8,9}$ plasticity effects, ${ }^{10}$ and alteration of cohesive energy. ${ }^{11}$ Two degradation mechanisms are primarily invoked to frame hydrogen effects in metals where hydride formation is not favorable and the material contains internal hydrogen: hydrogen-enhanced localized plasticity (HELP) and hydrogen-enhanced decohesion (HEDE). ${ }^{7}$ The HELP mechanism suggests that solute hydrogen atmospheres along the elastic strain field of a dislocation interact with those of neighboring dislocations, reducing the repulsive force between them and enhancing dislocation mobility. ${ }^{10-12}$ In this way, hydrogen-dislocation interactions lead to localized plasticity in regions of high hydrogen concentration and ultimately cause macroscopic brittle fracture via locally ductile processes. ${ }^{10}$ In contrast, observations of low-ductility fracture, 
occurring without significant plasticity in hydrogenexposed non-hydride-forming systems, ${ }^{11,13,14}$ led to the establishment of the HEDE mechanism. HEDE assumes that dissolved hydrogen weakens interatomic bonds at grain boundaries or other interfaces, and therefore lowers the cohesive energy, resulting in brittle failure at low applied stresses $^{13,14}$ and increased intergranular fracture in some systems. ${ }^{15,16}$ Additionally, recent investigations, such as those by Nagumo, ${ }^{17}$ point to the importance of vacancies in degradation processes. The hydrogen-enhanced strain-induced vacancy (HESIV) theory suggests that hydrogen enhances vacancy formation and agglomeration during deformation.

A wide variety of experimental and computational techniques can be applied to assess the dominant mechanisms influencing hydrogen effects on materials performance. For many years, conventional mechanical loading, such as quasistatic tensile/compression testing, ${ }^{18-22}$ cyclic deformation, ${ }^{23-26}$ and linear elastic fracture mechanics ${ }^{27-30}$ approaches, have been used to probe hydrogen-deformation interactions in myriad structural metals. These mechanical methods have been paired with numerous post-mortem characterization techniques ${ }^{22,31-33}$ to extract information about the importance of defects and interfaces on hydrogen diffusion and accumulation, to unravel hydrogen effects on crack nucleation and propagation, and to gain insight into the interplay between intrinsic material properties and hydrogen within the microstructure.

Several papers within this special topic are centered on coupling traditional test methods with novel characterization and analysis techniques to understand the chemical, mechanical, and microstructural factors that govern hydrogen-deformation interactions. The review article by Gibbs and coworkers surveys the existing literature to develop a method for using estimated intrinsic stacking fault energy (SFE) values, calculated with a sub-regular solution thermodynamic model, to compare the retention of tensile ductility of austenitic stainless steels in the presence of hydrogen. They demonstrate that intrinsic SFE is a highthroughput screening metric for a diverse range of austenitic stainless steel compositions with regard to hydrogen compatibility.

Harris, Thompson, and Burns employ a combination of multi-scale microscopy and diffraction techniques to assess the deformation induced proximate to the crack path by HEAC in a peak-aged Monel alloy. This approach leverages the strengths of three techniques: standard resolution electron backscatter diffraction (EBSD), high-resolution EBSD, and bright-field scanning transmission electron microscopy to build a multi-scale assessment methodology. The efficacy of such a methodology is highlighted, as are opportunities for additional advancements to develop clear linkages between microstructural elements and material resistance to HEAC.

Another novel method for inducing and characterizing hydrogen embrittlement has been presented in the article from Cheng and coworkers. They utilize digital image correlation to measure local strain and crack nucleation during tensile tests with simultaneous hydrogen charging. Their method is demonstrated on mooring chain steel, and they find that a transition from the HELP to the HEDE mechanism can explain the observed hydrogen embrittlement under different hydrogen charging configurations.

The advancement of three-dimensional atom probe tomography also enables position-sensitive, atomic-resolution characterization of low-atomicnumber (i.e. low-Z) elements. Such an approach, as presented in the article by Kim and coworkers, can be used to obtain significant insight into the role of grain boundary chemistry in hydrogen embrittlement. They find that carbon enrichment at grain boundaries in an austenitic steel enhances boundary cohesion, thus reducing intergranular fracture and hydrogen embrittlement.

Finally, nanoindentation has also been used to investigate hydrogen-deformation interactions in numerous materials. ${ }^{34-38}$ Nanoindentation is a powerful method for probing material response in the presence of hydrogen because it enables statistical analysis of properties measured with high fidelity in a small sampled area. ${ }^{35,39}$ Several studies have investigated hydrogen pre-charged metals, ${ }^{35,38,39}$ while others have focused on cathodically charging a material with hydrogen in situ. ${ }^{40-44}$ Ebner et al. implement electrochemical charging into a nanoindentation platform. Their electrochemical cell allows for nanoindentation experiments in liquid environments to specifically probe hydrogenmaterial interactions. The article demonstrates the measurement of hardness, modulus, and pop-in load in a nickel-based alloy, and evaluates the extent of hydrogen embrittlement.

The following list summarizes the papers being published under the topic of "Hydrogen Effects on Material Performance". To read or download the papers, follow the link http://link.springer.com/jour nal/11837/72/5/page/ 1 to the table of contents page for the May 2020 issue, vol. 72, no. 5 .

- P.J. Gibbs, P.D. Hough, K. Thürmer, B.P. Somerday, C. San Marchi, and J.A. Zimmerman. "Stacking Fault Energy Based Alloy Screening for Hydrogen Compatibility."

- Z.D. Harris, A.W. Thompson, and J.T. Burns. "Multiscale Assessment of Deformation Induced by Hydrogen Environment-Assisted Cracking in a Peak-Aged Ni-Cu Superalloy."

- X. Cheng, X. Zhang, Y. Wu, L. Wang, P. Zhao, and L. Yang. "The Character of Hydrogen Embrittlement in Mooring Chain Steel.” 
- K.-S. Kim, J.-H. Kang, and S.-J. Kim. "Effect of Carbon and Nitrogen on the Hydrogen Embrittlement of $15 \mathrm{Cr}-15 \mathrm{Mn}-4 \mathrm{Ni}$ Based Stable Austenitic Stainless Steels."

- A.S. Ebner, S. Brinckmann, E. Plesiutschnig, H. Clemens, R. Pippan, and V. Maier-Kiener. "A Modified Electrochemical Nanoindentation Setup for Probing Hydrogen-Materials Interaction Demonstrated on a Nickel-Base Alloy."

\section{REFERENCES}

1. C. Borchers, T. Michler, and A. Pundt, Adv. Eng. Mater. 10, 11 (2008).

2. Y. Murakami and H. Matsunaga, Int. J. Fatigue 28, 1509 (2006).

3. S. Fukuyama, M. Imade, Z. Lin, and K. Yokogawa, in: Proceedings of the ASME 2005 Pressure Vessels and Piping Conference Volume 6: Materials and Fabrication (Denver: ASME, 2005), pp. 493-497.

4. R.P. Gangloff and B.P. Somerday, Gaseous Hydrogen Embrittlement of Materials in Energy Technologies Volume 1: The Problem, Its Characterisation and Effects on Particular Alloy Classes (Philadelphia: Woodhead, 2012), p. ixx.

5. K.O. Findley, M.K. O’Brien, and H. Nako, Mater. Sci. Technol. 31, 1673 (2015).

6. L. Briottet, R. Batisse, P. Bernard, C. Duret-Thual, J.-L. Heuzé, F. Martin, F. Thebault, and F. Vucko, MechanicsMicrostructure-Corrosion Coupling, eds. C. Blanc and I. Aubert (Oxford: Elsevier; London: ISTE Press, 2019), p. 223.

7. W. Gerberich, Gaseous Hydrogen Embrittlement of Materials in Energy Technologies: Mechanisms, Modelling and Future Developments, Vol. 2, ed. R.P. Gangloff and B.P. Somerday (Philadelphia: Woodhead, 2012), pp. 209-246.

8. S.P. Lynch, Corros. Sci. 22, 925 (1982).

9. S. Lynch, Corros. Rev. 30, 105 (2012).

10. H.K. Birnbaum and P. Sofronis, Mater. Sci. Eng., A 176, 191 (1994).

11. R. Oriani and P. Joshephic, Acta Metall. 22, 1065 (1974).

12. Y. Jagodzinski, H. Hanninen, O. Tarasenko, and S. Smuk, Scr. Mater. 43, 245 (2000).

13. J. Kameda and C. McMahon, Metall. Mater. Trans. A 11, 91 (1980).

14. W.W. Gerberich and Y.T. Chen, Metall. Trans. A 6, 271 (1975).

15. S.M. Bruemmer, R.H. Jones, M.T. Thomas, and D.R. Baer, Metall. Mater. Trans. A 14, 223 (1983).

16. R.H. Jones, S.M. Bruemmer, M.T. Thomas, and D.R. Baer, Metall. Trans. A 13, 241 (1982).

17. M. Nagumo, Mater. Sci. Technol. 20, 940 (2004).

18. A.H. Windle and G.C. Smith, Met. Sci. J. 2, 187 (1968).

19. A. Kimura, Mater. Sci. Eng., A 58, 211 (1983).

20. Y. Yagodzinskyy, T. Saukkonen, S. Kilpeläinen, F. Tuomisto, and H. Hänninen, Scr. Mater. 62, 155 (2010).

21. D. Delafosse, Gaseous Hydrogen Embrittlement of Materials in Energy Technologies: Mechanisms, Modelling and Future
Developments, Vol. 2, ed. R.P. Gangloff and B.P. Somerday (Philadelphia: Woodhead, 2012), pp. 209-246.

22. S.K. Lawrence, Y. Yagodzinskyy, H. Hänninen, F. Tuomisto, E. Korhonen, Z.D. Harris, and B.P. Somerday, Acta Mater. 128, 218 (2017).

23. Y. Aoki, K. Kawamoto, Y. Oda, H. Noguchi, and K. Higashida, Int. J. Fract. 133, 277 (2005).

24. M. Nagumo, H. Shimura, T. Chaya, H. Hayashi, and I. Ochiai, Mater. Sci. Eng., A 348, 192 (2003).

25. P.J. Gibbs, C. San Marchi, K.A. Nibur, X. Tang, in: Proceedings of the ASME 2016 Pressure Vessels and Piping Conference Volume 6B: Materials and Fabrication (Vancouver: ASME, 2016) V06BT06A033.

26. Y. Takahashi, J. Sakamoto, M. Tanaka, K. Higashida, and H. Noguchi, Scr. Mater. 64, 721 (2011).

27. H.F. Jackson, K.A. Nibur, C. San Marchi, J.D. Puskar, and B.P. Somerday, Corros. Sci. 60, 136 (2012).

28. C. San Marchi, D.K. Balch, K. Nibur, and B.P. Somerday, J. Press. Vessel Technol. 130, 41401 (2008).

29. B.P. Somerday, M. Dadfarnia, D.K. Balch, K.A. Nibur, C.H. Cadden, and P. Sofronis, Metall. Mater. Trans. A Phys. Metall. Mater. Sci. 40, 2350 (2009).

30. K.A. Nibur, B.P. Somerday, D.K. Balch, and C.S. Marchi, Acta Mater. 57, 3795 (2009).

31. M.L. Martin, J.A. Fenske, G.S. Liu, P. Sofronis, and I.M. Robertson, Acta Mater. 59, 1601 (2011).

32. M.L. Martin, B.P. Somerday, R.O. Ritchie, P. Sofronis, and I.M. Robertson, Acta Mater. 60, 2739 (2012).

33. Z.D. Harris, J.J. Bhattacharyya, J.A. Ronevich, S.R. Agnew, and J.T. Burns, Acta Mater. 186, 616 (2020).

34. Y. Katz, N. Tymiak, and W.W. Gerberich, Eng. Fract. Mech. 68, 619 (2001).

35. K. Nibur, D. Bahr, and B. Somerday, Acta Mater. 54, 2677 (2006).

36. A. Basa, A. Barnoush, and C. Thaulow, Mech. Test. Diagnosis 2,5 (2012)

37. N. Kheradmand, J. Dake, and A. Barnoush, Philos. Mag. 92, 3216 (2012).

38. S.K. Lawrence, B.P. Somerday, N.R. Moody, and D.F. Bahr, JOM 66, 1383 (2014).

39. S.K. Lawrence, B.P. Somerday, and R.A. Karnesky, JOM 69,45 (2017).

40. A. Barnoush and H. Vehoff, Int. J. Mater. Res. 97, 1224 (2006).

41. A. Barnoush, J. Dake, N. Kheradmand, and H. Vehoff, Intermetallics 18, 1385 (2010).

42. A. Barnoush, N. Kheradmand, and T. Hajilou, Scr. Mater. 108, 76 (2015).

43. A. Barnoush and H. Vehoff, Scr. Mater. 55, 195 (2006).

44. M. Wen, A. Barnoush, and K. Yokogawa, Comput. Phys. Commun. 182, 1621 (2011).

Publisher's Note Springer Nature remains neutral with regard to jurisdictional claims in published maps and institutional affiliations. 